\title{
Differences in the endophytic fungal community and effective ingredients in root of three Glycyrrhiza species in Xinjiang, China
}

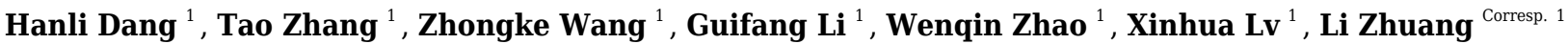 \\ ${ }^{1}$ College of Life Sciences, Shihezi University, Shihezi, Xinjiang, China \\ Corresponding Author: Li Zhuang \\ Email address: 3033573705@qq.com
}

\begin{abstract}
Background. Endophytic fungi influence the quality and quantity of the medicinal plant's bioactive compounds through specific fungus-host interactions. Nevertheless, due to the paucity of information, the composition of endophytic fungal communities and the mechanism by which effective ingredients regulate endophytic fungal communities in roots remains unclear.
\end{abstract}

Methods. In this study, we collected root and soil samples (depth range: 0-20, 20-40, and 40-60 cm) of three Glycyrrhiza species (Glycyrrhiza uralensis, Glycyrrhiza inflata, and Glycyrrhiza glabra). Glycyrrhizic acid and liquiritin content were determined using high-performance liquid chromatography (HPLC), and total flavonoid content was determined using ultraviolet spectrophotometry. High-throughput sequencing technology was employed to explore the composition and diversity of the endophytic fungal community in different root segments of three Glycyrrhiza species. Furthermore, soil samples were subjected to physicochemical analyses.

Results. We observed that the liquiritin content was not affected by the root depth $(0-20 \mathrm{~cm}, 20-40 \mathrm{~cm}$, and 40-60 cm). Still, it was significantly affected by the Glycyrrhiza species (Glycyrrhiza uralensis, Glycyrrhiza inflata, Glycyrrhiza glabra) ( $\mathrm{P}<0.05$ ). In Glycyrrhiza root, a total of 8 phyla and 140 genera were annotated so far, out of which Ascomycota and Basidiomycota phyla, and the Fusarium, Paraphoma, and Helminthosporium genera were found to be significantly dominant. Spearman correlation analysis revealed that liquiritin content was accountable for the differences in the diversity of the endophytic fungal community. Furthermore, distance-based redundancy analysis (db-RDA) showed that physicochemical properties of the soil (available potassium and ammonium nitrogen) and the root factors (liquiritin and water content) were the main contributing factors for the variations in the overall structure of the endophytic fungal community. Our results showed that the effective ingredients of Glycyrrhiza root and physicochemical properties of the soil regulated the endophytic fungal community composition and medicinal licorice diversity. 
1 Differences in the endophytic fungal community and

2 effective ingredients in root of three Glycyrrhiza

3 species in Xinjiang, China

4 Hanli Dang ${ }^{1}$, Tao Zhang ${ }^{1}$, Zhongke Wang ${ }^{1}$, Guifang Li ${ }^{1}$, Wenqin Zhao ${ }^{1}$, Xinhua Lv ${ }^{1}$, and Li

5 Zhuang ${ }^{1 *}$

$6{ }^{1}$ College of Life Sciences, Shihezi University, Shihezi city 832003, Xinjiang, China

7 Corresponding Author:

8 Li Zhuang ${ }^{1}$

$9{ }^{1}$ College of Life Sciences, Shihezi University, Shihezi city 832003, Xinjiang, China

10 Email address: 3033573705@qq.com

11 Abstract

Background. Endophytic fungi influence the quality and quantity of the medicinal plant's bioactive compounds through specific fungus-host interactions. Nevertheless, due to the paucity of information, the composition of endophytic fungal communities and the mechanism by which effective ingredients regulate endophytic fungal communities in roots remains unclear.

Methods. In this study, we collected root and soil samples (depth range: 0-20, 20-40, and 40$60 \mathrm{~cm}$ ) of three Glycyrrhiza species (Glycyrrhiza uralensis, Glycyrrhiza inflata, and Glycyrrhiza glabra). Glycyrrhizic acid and liquiritin content were determined using high-performance liquid chromatography (HPLC), and total flavonoid content was determined using ultraviolet spectrophotometry. High-throughput sequencing technology was employed to explore the composition and diversity of the endophytic fungal community in different root segments of three Glycyrrhiza species. Furthermore, soil samples were subjected to physicochemical analyses. Results. We observed that the liquiritin content was not affected by the root depth $(0-20 \mathrm{~cm}, 20$ $40 \mathrm{~cm}$, and 40-60 cm). Still, it was significantly affected by the Glycyrrhiza species (Glycyrrhiza uralensis, Glycyrrhiza inflata, Glycyrrhiza glabra) $(\mathrm{P}<0.05)$. In Glycyrrhiza root, a total of 8 phyla and 140 genera were annotated so far, out of which Ascomycota and Basidiomycota phyla, and the Fusarium, Paraphoma, and Helminthosporium genera were found to be significantly dominant. Spearman correlation analysis revealed that liquiritin content was accountable for the differences in the diversity of the endophytic fungal community. Furthermore, distance-based redundancy analysis (db-RDA) showed that physicochemical properties of the soil (available potassium and ammonium nitrogen) and the root factors (liquiritin and water content) were the main contributing factors for the variations in the overall structure of the endophytic fungal community. Our results showed that the effective ingredients of Glycyrrhiza root and 
34

35

36

37

38

39

40

41

42

43

44

45

46

47

48

49

50

51

52

53

54

55

56

57

58

59

60

61

62

63

64

65

66

67

68

physicochemical properties of the soil regulated the endophytic fungal community composition and diversity of medicinal licorices.

\section{Introduction}

Glycyrrhiza species are widely grown perennial herbs in arid and semi-arid regions (Zhang et al., 2005). Three Glycyrrhiza species stipulated in Chinese Pharmacopeia, namely dried root and rhizome of are Glycyrrhiza uralensis, Glycyrrhiza inflata, and Glycyrrhiza glabra. Its dried roots and rhizomes are widely used as herbal medicines in eastern and western countries (Rizzato, Scalabrin et al., 2017). A wide variety of effective ingredients, such as triterpene saponins, polysaccharides, and flavonoids (Tianshui Niu, 2009), are extracted from the roots of Glycyrrhiza (Wang, Yang et al., 2015). Glycyrrhizic acid, the chief triterpene saponin (Li-Ping, Cui-Ai et al., 2010), had demonstrated anti-inflammatory (Schröfelbauer, Raffetseder et al., 2009), antiviral, and immunoregulatory effects (Baba and Shigeta, 1987; Crance, Scaramozzino et al., 2003). Liquiritin is a major component of flavonoids that mainly exerts anti-inflammatory (Yina, a et al., 2018), antioxidant, and antibacterial effects (Weidner, Kordala et al., 2009; Antolak, Czyzowska et al., 2016). Due to its medicinal and economic value, the medicinal licorice plant has become a major research hotspot. The majority of the studies on licorice plants are focused on improving licorice content in licorice plants and discerning their ecological characteristics.

As per the conventional view, the quality and quantity of the bioactive compounds extracted from medicinal plants are primarily influenced by the genetic background of the medicinal plants, the ecological environment of the plant, and soil nutrients (Ncube, Finnie et al., 2012; Han, Jia et al., 2013). However, recent studies (S., H. et al., 2002; Huang, Cai et al., 2007; Shah, Rather et al., 2016; He, Cui et al., 2020) have shown that endophytic fungi substantially influences the quality and quantity of bioactive compounds in medicinal plants through specific fungus-host interactions.

Endophytes, in particular, endophytic fungi, are one of the most crucial components of plant's micro-ecosystems (Min, Ling et al., 2016). Endophytic fungi form a symbiotic relationship with its host plant, and it inhabits and grows in different healthy tissues of the host plant, including stems (Vaz, Mota et al., 2009), leaves (Hernawati, Wiyono et al., 2011), and roots (Radić, Likar et al., 2014). Endophytic fungi sequester carbohydrates and other nutrients from the host plant for its own growth (Singh and Mukerji, 2006) and, in exchange, confer multiple benefits to host plants. Endophytic fungi can promote the growth of host plants by increasing levels of growth hormones, such as gibberellin, indoleacetic acid, abscisic acid, and zeatin (Zhang, Wang et al., 1999). It also enhances the resistance of host plants to environmental stress by increasing the production of biologically bioactive compounds (Zhao, Shan et al., 2011; 
69 Ratnaweera, Silva et al., 2015). For instance, endophytic fungi promote plant growth and abiotic 70 stress resistance in wheat plants (Farhana, Alam et al., 2019). Besides, endophytic fungi increase 71 the accumulation of secondary metabolites, such as paclitaxel and deoxypodophyllotoxin in the 72 host plant (Firáková, Turdíková et al., 2007), thereby affecting the quantity and quality of 73 bioactive compounds of medicinal plants.

74 Endophytic fungi have demonstrated high biodiversity and are widely distributed in a 75 myriad of terrestrial and aquatic plants (Saikkonen, Faeth et al., 1998). Endophytic fungi were

76

77 78 79 80 isolated from multiple plants species, which includes important cash crop species (Pimentel, Glienke-Blanco et al., 2006), such as soybean, and medicinal plant species (Liu, Wenhong et al., 2017; Coutlyne, Thierry et al., 2018), such as Dendrobium officinale and Sceletium tortuosum. However, it is noteworthy that the rapid development of high-throughput sequencing technology and bioinformatics has enabled the identification of a plethora of novel fungal species (Taylor, Hollingsworth et al., 2014). Previous studies based on high-throughput sequencing technology have speculated that there are around 5.1 million fungal species, the majority of which are symbionts (Blackwell, 2011). Currently, only a small proportion of endophytic fungi could be isolated and identified, and the majority of the medicinal plant' endophytic fungi could not be cultured on routinely used media (Kivlin, Lynn et al., 2017). Therefore, it is indispensable to detect the endophytic fungal community in medicinal plants by adopting non-conventional culture methods. Modern molecular technology, specifically Illumina high-throughput sequencing technology, had comprehensively and accurately detected the diversity of endophytic fungal communities in medicinal plants (Berg, 2009; Kathrin, Blumenstein et al., 2015). Nextgeneration sequencing, a high-throughput sequencing technique, is a more robust and accurate characterization technique for the microbial community than $18 \mathrm{~S}$ rDNA-based non-culture methods and conventional culturing methods.

Numerous studies (Karliński, Rudawska et al., 2010) have shown that the host's genetic background (genotype or species) determines the composition of endophytic fungi. Soil fertility and the ecological environment, which directly affect the content of bioactive compounds in medicinal plants, showed indirect effects on the composition and structure of the endophytic fungal community (Min, Ling et al., 2016). However, so far, there is insufficient information on the composition of endophytic fungi in the root of medicinal licorice of different genetic backgrounds (species) and soil environmental factors that affect the community structure of endophytic fungi in the root of medicinal licorice plants. Thus, in this study, we investigated the distribution and composition of endophytic fungal species of three distinct medicinal licorices at three different root depths using high-throughput sequencing and explored their relationship with effective ingredients in the root of host plants and soil' physicochemical properties. The outcomes of this study will enhance researchers' understanding of the environmental and host 
105 factors that influence endophytic fungi and the symbiotic relationship between endophytic fungi

106 and medicinal plants. This study provides reference data for licorice growth for commercial and 107 medicinal use.

\section{Materials \& Methods}

109 Sample collection: The roots and rhizospheric soils samples (all samples were 0-20 cm, 20-40

$110 \mathrm{~cm}$, and 40-60 cm, respectively) of three Glycyrrhiza plants (Glycyrrhiza uralensis, Glycyrrhiza

111 inflata, and Glycyrrhiza glabra) were collected during August-September, 2019 from specimens

112 growing at three distinct sites in three different eco-regions of Xinjiang province, China. The

113 geographical location of sampling points and soil' physical and chemical properties are shown in

114 Table S1. To increase the statistical significance of the study we randomly selected three healthy

115 medicinal licorices plants from each geographical location as per the five-point sampling

116 method, and all root samples were cut with sterile scissors. The roots of each plant were divided

117 into three depth segments: upper $(0-20 \mathrm{~cm})$, middle $(20-40 \mathrm{~cm})$, and lower $(40-60 \mathrm{~cm})$. Roots of

118 each segment were equally divided into two parts: one part was used to determine the effective

119 ingredients of the Glycyrrhiza root samples and placed in a sterile plastic bag, and the second

120 part of the sample was put into a sterile bag and shipped to the laboratory in ice boxes for

121 microbial characterization. The soil and root materials from each eco-region were collected as

122 described above.

123 Surface sterilization: To remove the interference of other microbes, the surface of the licorice root was disinfected and sterilized in the laboratory as described previously (Saude, HurtadoGonzales et al., 2008). The samples from the final rinse solution were cultured using the potato dextrose agar (PDA) plate for $72 \mathrm{~h}$ at $28^{\circ} \mathrm{C}$. No fungal growth was observed on the PDA media, which suggested that root samples were effectively surface-sterilized (Cui, Vijayakumar et al., 2018). All root samples were labeled and immediately placed on ice and stored at liquid nitrogen until total DNA extraction.

Physicochemical analysis of the soil: For the physicochemical analysis of rhizospheric soil, the soil samples were air-dried and sieved ( $2 \mathrm{~mm}$ mesh), and the physicochemical analysis was performed as described previously by Bao et al. (Bao, 2008). Soil pH (soil: distilled water in 1: 5 ratio) was measured using a $\mathrm{pH}$ meter, and soil water content (SWC) was measured by weighing. The content of organic matter (SOM) and total salt (TS) were measured by external heating with potassium dichromate and atomic absorption spectrometry, respectively. The total nitrogen (STN), total phosphorus (STP), and total potassium (STK) content were determined by the acid digestion method. $0.01 \mathrm{M}$ calcium chloride extraction method was used to determine the soil nitrate-nitrogen (SNN) and soil ammonium nitrogen (SAN) levels. The available phosphorus (SAP) content was measured by the sodium bicarbonate extraction method (molybdenum- 
140 antimony colorimetry). The available potassium (SAK) content was determined by the

141 ammonium acetate extraction method using atomic absorption spectrometry.

142 Determination of effective ingredients of Glycyrrhiza plant root: The Glycyrrhiza root

143 samples were dried to constant weight, powdered using mortar and pestle, and sieved through

14460 -mesh. To analyze the effective ingredients, $0.2 \mathrm{~g}$ of sieved root powder samples were

145 extracted using chromatographic methanol (71\% concentration) in the ultrasonic bath (250 W, 40

$146 \mathrm{kHz}$ ). The levels of effective ingredients, i.e., glycyrrhizic acid (GIA) and liquiritin (LI), were

147

148

149

150

151

152

153

154

155

156

157

158

159

160

161

162

163

164

165

166

167

168

169

170

171

172

173

174

175 measured by high-performance liquid chromatography (HPLC, Agilent-1260 Infinity, USA), as described previously (Dang, Zhang et al., 2020). Agilent ZORBAX SB-C18 column $(150 \mathrm{~mm} \times 4.6 \mathrm{~mm}, 5 \mu \mathrm{m})$, DAD detector, and a mobile phase (chromatographic methanol: ultrapure water: $36 \%$ glacial acetic acid $=71: 28: 1$; acetonitrile: $0.5 \%$ glacial acetic acid $=1: 4 ; 5 \mu \mathrm{L}$ injection volume; $1.0 \mathrm{~mL} \cdot \mathrm{min}-1$ elution rate) were used in the HPLC analysis. For calibration purposes, the reference materials of GIA and LI were: (CAS\#1405-86-3) and LI (CAS\#551-155), respectively, from Solarbio. The total flavonoid (GTF) content in root was measured by ultraviolet spectrophotometry at $334 \mathrm{~nm}$ with the liquiritin standard (CAS\#551-15-5) from Solarbio as the control.

DNA extraction and library construction: Total genomic DNA was extracted from $0.5 \mathrm{~g}$ of root samples using the DNA Quick Plant System kit (Tiangen, China) as per the manufacturer's protocol. The concentration and integrality of extracted DNA were detected using a NanoDrop2000 (Thermo Fisher Scientific, USA) and 1\% agarose gel electrophoresis, respectively. After determining the final concentration, DNA samples were diluted to $1 \mathrm{ng} / \mu \mathrm{L}$ with sterile distilled water, and each PCR product was used as template DNA. The ITS (Internal Transcribed Spacer) rDNA genes of the ITS1 region were amplified using specific primers (ITS5-1737F 5'-GGAAGTAAAAGTCGTAACAAGG-3'and ITS2-2043R 5'-

GCTGCGTTCTTCATCGATGC-3') with barcodes (David, Berry et al. 2011). To ensure amplification efficiency and accuracy, all PCR reactions were performed with Phusion ${ }^{\circledR}$ HighFidelity PCR Master Mix and GC Buffer (New England Biolabs). The temperature regime for PCR reactions was as follows: $95^{\circ} \mathrm{C} / 3 \mathrm{~min}, 30$ cycles $\left(95^{\circ} \mathrm{C} / 30 \mathrm{~s}, 55^{\circ} \mathrm{C} / 30 \mathrm{~s}, 72^{\circ} \mathrm{C} / 30 \mathrm{~s}\right)$, and $72^{\circ} \mathrm{C} / 5 \mathrm{~min}$. PCR products were mixed with $1 \mathrm{X}$ loading buffer (containing SYBR green) in equidensity ratios and visualized on $2 \%$ agarose gel electrophoresis. The target sequences were purified using GeneJET ${ }^{\mathrm{TM}}$ Gel Extraction Kit (Thermo Scientific). The DNA libraries were constructed using TruSeq ${ }^{\circledR}$ DNA PCR-Free Sample Preparation Kit (Illumina, USA) as per the manufacturer's instruction, and the quality was assessed on the Qubit ${ }^{\circledR} 2.0$ Fluorometer (Thermo Scientific) and Agilent Bioanalyzer 2100 system. ITS sequencing was carried out with the Illumina platforms (HiSeq2500) at the Beijing Compass Biotechnology Co., Ltd. (Beijing, China). 
176 Bioinformatics analysis and statistical analysis: Cutadapt (Liu, Zhenshan et al., 2016)

177

178

179

180

181

182

183

184

185

186

187

188

189

190

191

192

193

194

195

196

197

198

199

200

201

202

203

204

205

206

207

208

209

210

software was employed to assign the single-end reads to the respective samples based on the unique barcode, and single-end reads were truncated by cutting off the barcodes and primer sequences. Before the subsequent analysis, a total of 2,199,148 raw sequences were filtered by using Cutadapt software to remove the influence of the non-microbiota community, including chloroplast and mitochondrial sequences. Cutadapt software specific filtering conditions were used for strict quality control in order to generate high-quality clean reads. Clean reads were obtained by comparison with the reference database (Unite database) (Haas, Gevers et al., 2011) using the UCHIME algorithm to detect and remove chimeric sequences.

UPARSE software (Martin, 2011) (Version 7.0.1001) was used to cluster the clean reads into the same operational taxonomic units (OTUs) with $\geq 97 \%$ similarity. The clean reads with the highest frequency were used as the representative sequence of each OTU. The classification information for each representative sequence was annotated through the Unite database based on the BLAST algorithm using QIIME software (Version 1.9.1). To decipher the phylogenetic relationship among 27 samples, MUSCLE (Version 3.8.31) software was employed for multiple sequence alignment. The OTU abundance information was normalized by the standard sequence number corresponding to the minimum sequence sample $(54,262$ reads for sample D.2.1).

Alpha diversity analysis based on output normalized data were used to study the complexity of species diversity in a sample using six indices (observed-species, Shannon, Simpson, Chao1, ACE, and good-coverage) (Li, Zhang et al., 2013). All indices in the samples were calculated with QIIME (Version 1.7.0) and displayed with R software (Version 3.6.1).

To evaluate differences in sample species complexity, the beta diversity analysis of outputnormalized data was used, which was based on weighted Unifrac and calculated using QIIME software. The Un-weighted Pair-group Method with Arithmetic Mean (UPGMA) clustering analysis was conducted by QIIME software (Version 1.7.0). In addition, R software (Version 3.6.1) was also used for rarefaction curve generation, Wilcoxon rank-sum test, Metastat statistical test, Spearman correlation analysis of heat maps, and Distance-based Redundancy Analysis (db-RDA). Pearson correlation analysis (Pearson coefficient, $r$ ) was performed for the effective ingredients and the physicochemical properties of the soil, with the significance level set to 0.05. ANOVA was performed with SPSS (Version19.0) (IBM Inc., Armonk, USA) and displayed with GraphPad Prism 5. The statistically significant differences were determined by ANOVA, followed by Bonferroni's statistical test for multiple comparisons, and the significance level was set to 0.05 .

\section{Results}

\section{Differences in levels of effective ingredients in Glycyrrhiza roots}


211 The effective ingredients of Glycyrrhiza roots and physicochemical properties of soil are 212 presented in Table S2. The results of two-way ANOVA showed that the levels of the effective 213 ingredients, i.e., glycyrrhizic acid (GIA), liquiritin (LI), and total flavonoid (GTF), were not 214 significantly affected by the interaction between root depth $(0-20 \mathrm{~cm}, 20-40 \mathrm{~cm}$, and $40-60 \mathrm{~cm})$ 215 and plant species (Glycyrrhiza uralensis, Glycyrrhiza inflata, and Glycyrrhiza glabra) $(\mathrm{P}>0.05)$ 216 (Table S3). However, the content of LI was significantly affected by the main effect plant species 217 ( $<<0.05)$ (Table S3 and Figure 1). As shown in Figure 1, LI content in Glycyrrhiza uralensis $218(\mathrm{Gu})$ root was significantly higher than Glycyrrhiza inflata $(\mathrm{Gi})(\mathrm{P}<0.05)$ and Glycyrrhiza 219 glabra $(\mathrm{Gg})$ roots $(\mathrm{P}<0.05)$ (Figure 1a).

220 As per the Pearson correlation analysis, levels of effective ingredients were significantly 221 correlated with the physicochemical properties of the soil (Table 1). GIA content in Glycyrrhiza 222 level was significantly and positively correlated to the available potassium (SAK) and water 223 content of the soil (SWC) $(r>0 ; \mathrm{P}<0.05)$; however, LI level in root was significantly and 224 negatively correlated to SAK and total salt (TS) content of the soil $(\mathrm{r}<0 ; \mathrm{P}<0.05)$.

225

226

227

228

229

230

231

232

233

234

235

236

237

238

239

240

241

242

243

244

245

246

\section{Sequencing of Glycrrhiza root's endophytic fungi}

A total of 2,118,633 effective sequences were identified by sequencing the root samples from three Glycyrrhiza (Glycyrrhiza uralensis, Glycyrrhiza glabra, and Glycyrrhiza inflata) species using Illumina HiSeq sequencing and after filtering out low-quality and short sequence reads. The sequencing results of each sample are listed in Supplementary Table S4. The effective sequences were clustered into OTUs with 97\% identity, and a total of 1,063 OTUs were obtained. Out of the total effective sequences, $91.53 \%$ were assigned to the kingdom level, $59.27 \%$ to the phylum level, $54.37 \%$ to the class level, $53.72 \%$ to the order level, $46.19 \%$ to the family level, $38.01 \%$ to the genus level, and $23.52 \%$ to the species level (Figure S1a). The rarefaction curves showed that the number of OTU in each sample increased gradually with the sequence quantity, which validated that the sequencing data was adequate for the analysis (Figure S1b).

\section{Differences in alpha diversity}

The alpha diversity index of each group is demonstrated in Table S5. Alpha diversity indices, Shannon and Chao1, deciphered the diversity and richness of microbial communities in Glycyrrhiza root samples. A higher index value denotes higher species diversity and distribution. The Shannon index of the Gu1 (4.910) sample was the highest, and that of the Gi1 (3.393) sample was the lowest. Moreover, the Gi1 root sample showed the lowest Chao1 (238.678) and ACE (253.105) values, while the Gi3 root sample showed the highest Chao1 (356.317) and ACE (355.694) values. As per Wilcoxon rank-sum analysis, the Shannon index showed significantly different distribution between Gu and Gi samples, especially at 0-20 cm at the root depth (Figure $2 a)$. The Shannon index in the Gul root sample was significantly higher than the Gi1 root sample $(\mathrm{P}<0.05)$. Furthermore, the Chao1 index in sample Gi increased gradually with decreasing root 
247 depths, and as per the Wilcoxon rank-sum test, sample Gi'Chaol index value was significantly

248 affected by root depth (Figure 2b). Specifically, the Chaol index value of Gi3 sample was

249 significantly higher than the Gi1 sample $(\mathrm{P}<0.01)$, and that of the Gi2 root sample was

250 significantly higher than the Gi1 root sample $(\mathrm{P}<0.05)$.

251 Differences in beta diversity

252 To evaluate differences in microbial community composition among Glycyrrhiza root 253 samples, beta diversity analysis was performed. The Unweighted Pair-Group Method with 254 Arithmetic (UPGMA) cluster analysis was performed to discern similarity in the composition of 255 endophytic fungal community among different Glycyrrhiza root samples. The UPGMA 256 clustering results were integrated with the relative abundance of species at the phyla and taxon 257 levels in each group. The UPGMA cluster tree outcomes based on Weighted Unifrac distances 258 showed that Gg3, Gg2, Gg1, Gu1 samples and Gu3, Gi2, Gu2, Gi1 samples were clustered 259 together (Figure 3a). Meanwhile, to discern the difference in the beta diversity between different 260 groups of samples, a Wilcoxon rank-sum test based on Weighted Unifrac distances was 261 262 263 264 265 266 267 268 269

270

271

272

273

274

275

276

277

278

279

280

281 constructed (Figure 3b). The outcomes of this test showed that there were significant differences in beta diversity between the Gu and Gi group of samples, which was consistent with the UPGMA cluster tree. Specifically, there were significant differences in beta diversity between Gi1 and Gi2 samples $(\mathrm{P}<0.05)$, Gi3 and Gu3 samples $(\mathrm{P}<0.05)$, and $\mathrm{Gi} 1$ and $\mathrm{Gu} 1$ samples $(\mathrm{P}<$ 0.01 ) (Figure 3b). It indicated that endophytic fungal community composition differed significantly between different species and different root depths in medicinal licorice plants.

\section{Differences in endophytic fungal community composition in medicinal licorice}

Based on OTU sequences and the Unite database, total sequences were annotated into 8 phyla, 23 classes, 53 orders, 102 families, 140 genera, and 141 species. The most abundant endophytic fungal phyla in all the nine groups are enumerated in Figure 4a. Ascomycota phyla were found to be the most dominant phyla among all the samples, accounting for $91.821 \%$, $60.558 \%, 39.956 \%, 79.651 \%, 62.305 \%, 54.241 \%, 82.176 \%, 81.928 \%$, and $80.290 \%$ of the total number of species in Gi1, Gi2, Gi3, Gg1, Gg2, Gg3, Gu1, Gu2, and Gu3 samples, respectively. In addition, Basidiomycota accounted for $21.348 \%, 28.440 \%, 10.631 \%, 12.523 \%, 6.749 \%$, and $5.110 \%$ of relative abundance in $\mathrm{Gi} 2, \mathrm{Gi} 3, \mathrm{Gg} 2, \mathrm{Gg} 3, \mathrm{Gu} 2$, and Gu3 samples, respectively. The relative abundance of Ascomycota phyla decreased with increasing root depth. To determine the differences at the phylum level in different groups of root samples, a MetaStat statistical test based on species abundance was conducted. As per the outcomes of this test, the relative abundance of Ascomycota in sample Gi showed significant differences in distribution at different root depths (Figure 4b). Specifically speaking, the relative abundance of Ascomycota in the Gi1 sample (91.821\%) was significantly higher than the Gi3 sample (39.956\%) (Figure 4b). 
282

283

284

285

286

287

288

289

290

291

292

293

294

295

296

297

298

299

300

301

302

303

304

305

306

307

308

309

310

311

312

313

314

315

At genus level, the top 10 dominant fungal genera based on relative abundance in each group (Figure 4c) were Fusarium (Gi1: 27.907\%, Gg1: 23.944\%, Gg2: 31.071\%, Gg3: 25.381\%, Gu1: 19.253\%, Gu3: 18.215\%), Paraphoma (Gi1: 27.738\%, Gi3: 23.937\%, Gu3: 13.980\%), Helminthosporium (Gi1: 26.567\%, Gg1: 25.124\%, Gu1: 8.224\%, Gu2: 17.408\%), Sarocladium (Gi2: 3.326\%, Gg1: 16.547\%, Gg2: 17.243\%, Gg3: 21.897\%, Gu1: 4.218\%), Cladosporium (Gi2: 6.446\%, Gi3: 2.721\%, Gu3: 15.174\%). Furthermore, Cadophora (13.200\%) and Psathyrella (10.917\%) were found to be the most dominant genera in Gi2 sample, Tomentella (14.472\%) in Gi3 sample, and Conocybe (12.068\%) in Gg3 sample (Figure 4c).

At the same time, details of the composition of the top 10 dominant fungi at other classification levels (Class, Older, Family and Species) were listed in Table S6. Specifically speaking, Sordariomycetes, Dothideomycetes, Agaricomycetes were found to be the dominant class; Hypocreales, Pleosporales, Thelephorales dominant order; Nectriaceae, Phaeosphaeriaceae, Massarinaceae dominant family; Fusarium-solani, Paraphoma-radicina, Sarocladium-kiliense dominant species.

\section{Correlation of effective ingredients with physicochemical properties of soil and endophytic} fungal community in the Glycyrrhiza roots

Spearman correlation analysis showed that the LI content was significantly and positively correlated with the alpha diversity index $(\mathrm{r}>0, \mathrm{P}<0.05)$ (Figure 5). Besides, LI content showed a highly significant and positive correlation with the Shannon index, Simpson index, and Chao1 index $(\mathrm{P}<0.05)$. It indicated that the LI content led to the differences in the diversity of the endophytic fungal community in medical licorice roots in this study.

Distance-based redundancy analysis (db-RDA) based on the Bray-Curtis distance showed that the effective ingredients and physicochemical properties of the soil had significant effects on the differences in the endophytic fungal community (Figure 6). The differential distribution of endophytic fungal community was restricted primarily to the first and second ordination axes and explained $16.23 \%, 13.89 \%$ of the total variability, respectively (Figure 6). Out of all the soil' environmental factors, SAK content affected the differences of the endophytic fungal community most significantly $\left(\mathrm{r}^{2}=0.329, \mathrm{P}<0.01\right)$, followed by SAN $(\mathrm{P}<0.05)$. Among the root factors, RWC most significantly affected the difference of endophytic fungal communities $\left(\mathrm{r}^{2}=0.247, \mathrm{P}\right.$ $<0.05$ ), followed by LI content $(\mathrm{P}<0.05)$ (Figure 6, Table S7). As per the outcomes of the dbRDA analysis, the SAN, SAK, RWC, and LI content were the major factors contributing to the variations in the overall structure of the endophytic fungal community in the roots of the medicinal plants in the current study.

\section{Discussion}

Peer] reviewing PDF | (2020:11:55159:3:0:NEW 3 Feb 2021) 
316

317

318

319

320

321

322

323

324

325

326

327

328

329

330

331

332

333

334

335

336

337

338

339

340

341

342

343

344

345

346

347

348

349

350

351

In the current study, the composition and diversity of endophytic fungal communities at different root depth range $(0-20 \mathrm{~cm}, 20-40 \mathrm{~cm}$, and 40-60 cm) of three Glycyrrhiza species (Glycyrrhiza uralensis, Glycyrrhiza glabra, and Glycyrrhiza inflata) were investigated using high-throughput sequencing technology. Thus, a highly accurate and substantial amount of data was procured than previous studies based on conventional technology (Tie, Hu et al., 2010; Jiang-Tao, Xiao-Xia et al., 2013; Palak, Arora et al., 2019). As per the alpha and beta diversity analysis of endophytic fungal community, fungal communities between Glycyrrhiza uralensis and Glycyrrhiza inflata showed significant differences at different root depths $(0-20 \mathrm{~cm}, 20-40$ $\mathrm{cm}$, and 40-60 cm) (Figure 2 and Figure 3). It indicated that the host plant's genotype and ecological region contributed to the differences in endophytic fungal communities. Numerous studies (Saikkonen, Wäli et al., 2004) have demonstrated that the adaptation of the endophytic fungal community primarily relies on adapting host plants to the ecological environment. It implies that host plants substantially influence the colonization and distribution of the endophytic fungal communities. The interaction between fungus and host plant is often considered dynamic where orientation is determined by subtle differences in the expression of fungal genes in response to the host or, conversely, by the host's recognition and response fungus. Thus, slight genetic differences in the two genomes control the symbiosis (Moricca and Ragazzi, 2008).

Furthermore, the current study also showed that root depth significantly affected the richness and composition of the endophytic fungal community (Figure 2 and Figure 3). It indicated that ecologically different fungi might represent certain ecological regions (root depth). It might be a crucial factor that should be taken into account while inoculating endophytic fungi into the host plants. We speculated that this could be correlated to root respiration and soil C content. Root respiration, which accounts for $60 \%$ of total soil respiration, regulates the metabolism of roots and soil microbes and is considered a significant contributor to the terrestrial carbon budget (Pregitzer, Laskowski et al., 1998). Also, the C content in unstable soil varies significantly at different soil depths (de Graaff, Jastrow et al., 2014). Moreover, Noah Fierer et al. (Fierer, Schimel et al., 2003) demonstrated that the vertical distribution of the specific microbial species was correlated mainly to decreased carbon availability with increasing soil depth.

In this study, we employed high-throughput sequencing to determine the composition of endophytic fungal communities at different taxonomic levels (phylum, class, older, family, genus, and species) (Figure 4a, Figure 4c, and Table S6). The results indicated that 27 samples of medicinal licorice roots contained a peculiar microbiome. For example, Ascomycota was the dominant phylum in all samples, followed by Basidiomycota, which was in line with previous studies (Stephenson, Tsui et al., 2013; Tan, Zhou et al., 2018). The phylum Ascomycota, the largest phylum of fungi, entails a highly diverse population and plays a vital role in genetics

Peer] reviewing PDF | (2020:11:55159:3:0:NEW 3 Feb 2021) 
352 (Wallen and Perlin, 2018), ecology (Belnap and Lange, 2005), and phylogeny (López-Giráldez, 353 Crous et al., 2009). For instance, Ascomycota produces large numbers of spores through both

354

355

356

357

358

359

360

361

362

363

364

365

366

367

368

369

370

371

372

373

374

375

376

377

378

379

380

381

382

383

384

385

386

387 asexual and sexual reproduction. To disperse ascospores, asci act as small water cannon and sprays spores into the air. Spores are also spreads multiple phytopathogenic and saprophytic fungi (Trail, 2010). Most of the members of Ascomycota are saprophylaxis and plays an important role in organic matter decomposition in the soil. In this process, the dominant fungal community assimilates root exudates for organic matter degradation (Mylène, Patricia et al., 2018). He et al. (He, Cui et al., 2020) showed that inoculation of licorice plants with darkcolored septogenic endophyte (DSE), conidia, or sterile ascomycetes increased root biomass, uptake of nitrogen $(\mathrm{N})$ and phosphorus $(\mathrm{P})$ by roots, and concentration of glycyrrhizin and glycyrrhizic acid.

Moreover, outcomes of this study indicated that the relative abundance of Ascomycota gradually decreased with the increasing root depths (Figure 4b), in line with the previous study by Ko, Daegeun et al. (Ko, 2015). Based on this data, we investigated the correlation between Ascomcycota in Glycyrhizza roots and root depth. We observed that the relative abundance of Ascomycota in Glycyrrhiza inflata differed significantly with root depth. However, the relative abundance of Ascomycota in Glycyrrhiza uralensis and Glycyrrhiza glabra did not differ significantly with root depth. It indicated that specific endophytes proliferate preferentially in certain ecological regions and play different ecological roles than other endophytes. Concisely, in addition to soil depth, the relative abundance of endophytes was also correlated to the genotype of the host plant species. These findings were in line with the study on host genotype and soil conditions on the ectomycorrhizal community of poplar clones (Karliński, Rudawska et al., 2013).

However, the primary limitation of the generalization of the current study's outcomes is that three samples of Glycyrrhiza plant roots were collected from distinct geographical areas. As it is challenging to procure three distinct Glycyrrhiza species from the same habitat, to a certain extent, physicochemical properties of the soil can represent the environmental factors of Glycyrrhiza species. Therefore, in this study, we investigated the effect of the root as well as soil factors.

Numerous studies (Da-Cheng, Hao et al., 2015; Li and Wu, 2018) reported that the accumulation of effective ingredients in medicinal licorice roots is affected by multiple factors. In this study, the LI content was affected more by the plant species than root depth (Table S3). LI content in Glycyrrhiza uralensis root was significantly higher than in Glycyrrhiza inflata and Glycyrrhiza glabra (Figure 1a), in line with a previous study (Zhang, Yang et al., 2018). We speculate that it might be correlated to the expression of specific functional genes, which might be strongly correlated to the content of effective ingredients, such as glycyrrhizic acid and 
388 liquiritin in the root of the licorice species. As per the previous studies (Winkel-Shirley 2002, 389 2017; Mochida, Sakurai et al., 2017), key functional genes, such as chalcone synthase gene, 3390 Hydroxy-3-methylglutary CoA reductase (HMGR), and squalene synthase (SQS), regulate the

391

392

393

394

395

396

397

398

399

400

401

402

403

404

405

406

407

408

409

410

411

412

413

414

415

416

417

418

419

420

421

422

transcription of glycyrrhizic acid and liquiritin. However, further in-depth analysis is required to characterize the expression of functional genes of effective ingredients. The current study provides a theoretical basis for the developmental strategies related to the improvement of Glycyrrhiza uralensis cultivation. The content of effective ingredients in Glycyrrhiza root samples may result from the interaction between plants and their environment during plant growth. Thus, the accumulation of effective ingredients in root is influenced by its ecological environment. In this study, GIA, GTF, and LI content showed a positive correlation with soil total nitrogen $(\mathrm{STN})(\mathrm{r}>0)$ (Table 1). Thus, we speculate that the majority of the soil nutrients can promote the accumulation of effective ingredients; however, certain soil nutrients, such as soil total potassium (STK), are an exception. Potassium activates multiple enzyme systems and increases stress resistance in plants (Wang and $\mathrm{Wu}, 2017$ ). In this study, STK was found to be negatively correlated $(r<0)$ to the GIA, GTF, and LI content, in line with the previous study (Liu, Li et al., 2020). In addition, soil available potassium (SAK) was found to be significantly and positively correlated to GIA level but significantly and negatively correlated to LI level (Table 1). In this study, we speculated that the utilization mechanism of soil nutrients by effective ingredients might be entirely different. However, the underlying mechanism for potassium mediated regulation of effective ingredients remains unclear. Thus, this study may provide platform data for an in-depth analysis. In general, these soil factors exhibit habitatspecific characteristics for regulating the effective ingredients in licorice roots.

In recent years, a growing number of studies (Stegen, Lin et al., 2012; Edwards, Johnson et al., 2015; Nuccio, Anderson-Furgeson et al., 2016) reported that the dynamics of the microflora is driven to a large extent by environmental factors, such as soil characteristics ( $\mathrm{pH}$, nitrogen, phosphorus, and potassium) and climatic conditions (rainfall and temperature). In line with these reports, our study showed that LI, RWC, SAN, and SAK content were the major contributing factors to the variations in the overall structure of the endophytic fungal community (Figure 6 and Table S7). In addition, we found that the LI content in Glyrrhiza root was significantly and positively correlated to the diversity of endophytic fungal community (Shannon and Simpson index) $(\mathrm{P}<0.05)$ (Figure 6). Liquiritin (LI), an essential component of flavonoids, confers clinical efficacy to the medicinal licorices and serves as an important quality index for determining the quality of medicinal licorices. Flavonoids synthesis in host plants is induced when the symbiotic fungus is acted upon by purified signaling molecules secreted from the same fungal cells during colonization. Chen et al. (Meilan, Chen et al., 2017) demonstrated that stem

Peer) reviewing PDF | (2020:11:55159:3:0:NEW 3 Feb 2021) 
423 biomass, root biomass, and liquiritin content in the root of host plants increased significantly 424 when inoculated with fungi Glomus mosseae, Glycyrrhiza uralensis.

425 Meanwhile, our results showed that physicochemical factors of the soil and effective 426 ingredients had a significant effect on the composition of endophytic fungal communities. It 427 demonstrated the interaction between endophytic fungal community, root factors, and soil 428 factors. Thus, it indicated that fungal composition could be altered by altering soil factors (Liu, 429 Jinshan et al., 2018), promoting the accumulation of effective ingredients in plants (Han, Jia et 430 al., 2013). Wei Xie et al. (Xie, Hao et al., 2019) showed that in medicinal licorice, P addition and 431 arbuscular mycorrhizal (AM) inoculation improved plant growth and facilitated glycyrrhizic acid 432 and liquiritin accumulation in Glycyrrhiza uralensis. Meanwhile, Y. Orujei et al. (Orujei, 433 Shabani et al., 2013) also showed arbuscular mycorrhizal fungi (AMF) inoculation enhanced the growth rate and accumulation of effective ingredients in licorice roots (Glycyrrhiza glabra) as compared to control.

In general, the current study unraveled the ecological role of non-biological factors (soil and root) in the endophytic fungal community composition of medicinal licorices. Besides, this study provides crucial information for the developmental strategies related to improving the production and quality of medicinal licorice plants. However, further in-depth studies are required to characterize the functions of the endophytic fungi.

441

442

443

444

445

446

447

448

449

450

451

452

453

454

455

456

\section{Acknowledgements}

In this study, we would like to thank professor L.Z for hers guidance, all the authors for their joint efforts. We also would like to thank many graduate students and staff who directed the collection of soil samples that were not listed as co-authors.

\section{References}

Antolak H, Czyzowska A, Kregiel D. 2016. Antibacterial and Antiadhesive Activities of Extracts from Edible Plants against Soft Drink Spoilage by Asaia spp. Journal of food protection 80:25-34.

Baba M, Shigeta S. 1987. Antiviral activity of glycyrrhizin against varicella-zoster virus in vitro. Antiviral Research 7:99-107.

Bao S. 2008. Soil Agro-chemistrical Analysis. China Agriculture Press 22-196.

Belnap J, Lange OL. 2005. Lichens and microfungi in biological soil crusts: community structure, physiology, and ecological functions. MYCOLOGY SERIES 23:117.

Berg G. 2009. Plant-microbe interactions promoting plant growth and health: perspectives for controlled use of microorganisms in agriculture. Applied Microbiology \& Biotechnology 84:11-18. 
457 Blackwell, M. 2011. The Fungi: 1, 2, 3 ... 5.1 million species?, 98:426-438.

458 Coutlyne MM, Thierry R, Ajay K, Cornelius BC, Njie AC.2018. Phylogenetic analysis and

459

460

461

462

463

464

465

466

467

468

469

470

471

472

473

474

475

476

477

478

479

480

481

482

483

484

485

486

487

488

489

490

491 diversity of novel endophytic fungi isolated from medicinal plant Sceletium tortuosum. Phytochemistry Letters 27:36-43.

Crance JM, Scaramozzino N, Jouan A, Garin D. 2003. Interferon, ribavirin, 6-azauridine and glycyrrhizin: antiviral compounds active against pathogenic flaviviruses. Antiviral Res 58:73-79.

Cui, J. L., Vinod V, Gang Z. 2018. Partitioning of Fungal Endophyte Assemblages in RootParasitic Plant Cynomorium songaricum and Its Host Nitraria tangutorum. Frontiers in Microbiology 9:666.

Da-Cheng, Hao, Pei-Gen, Xiao. 2015. Genomics and Evolution in Traditional Medicinal Plants: Road to a Healthier Life. Evolutionary Bioinformatics Online 11: EBO. S31326.

Dang H, Zhang T, Li G, MU Y, Lv X, Wang Z, Zhuang L. 2020. Root-associated endophytic bacterial community composition and structure of three medicinal licorices and their changes with the growing year. BMC microbiology 20: 1-18.

David, Berry, Ben Mahfoudh K, Wagner M, Loy A. 2012. Barcoded Primers Used in Multiplex Amplicon Pyrosequencing Bias Amplification. Applied \& Environmental Microbiology 78:612-612.

de Graaff, M.-A., J. D. Jastrow, S. Gillette, A. Johns and S. D. Wullschleger. 2014. Differential priming of soil carbon driven by soil depth and root impacts on carbon availability. Soil Biology \& Biochemistry 69: 147-156.

Edwards J, Johnson C, Santos-Medellín C, Lurie E, Sundaresan V. 2015. Structure, variation, and assembly of the root-associated microbiomes of rice. Proceedings of the National Academy of ences of the United States of America 112: E911-E920.

Farhana, Alam, Ripa, Wei-Dong, Cao, Shuai, Tong, Jian-Guang, Sun. 2019. Assessment of Plant Growth Promoting and Abiotic Stress Tolerance Properties of Wheat Endophytic Fungi. Biomed Research International 2019: 6105865.

Fierer N, Schimel JP, Holden PA. 2003. Variations in microbial community composition through two soil depth profiles. Soil Biology \& Biochemistry 35:167-176.

Firáková, S., M. Šturdíková and M. Múčková. 2007. Bioactive secondary metabolites produced by microorganisms associated with plants. Biologia 62: 251-257.

Haas, B. J., D. Gevers, A. M. Earl, M. Feldgarden, D. V. Ward, G. Giannoukos, D. Ciulla, D. Tabbaa, S. K. Highlander and E. Sodergren. 2011. Chimeric 16S rRNA sequence formation and detection in Sanger and 454-pyrosequenced PCR amplicons. Genome research 21: 494-504. 
492

493

494

495

496

497

498

499

500

501

502

503

504

505

506

507

508

509

510

511

512

513

514

515

516

517

518

519

520

521

522

523

524

525

Han T, Jia M, Lu B, Qin L. 2013. Relationships between endophytic fungi and their hosts: impacts on drug quality of medicinal plants. Planta Medica 79: 1256.

He, C., J. Cui, X. Chen, W. Wang and J. Hou. 2020. Effects of enhancement of liquorice plants with dark septate endophytes on the root growth, glycyrrhizic acid and glycyrrhizin accumulation amended with organic residues. Current Plant Biology: 100154.

Hernawati H, Wiyono S, Santoso S. 2011. Leaf endophytic fungi of chili (Capsicum annuum) and their role in the protection against Aphis gossypii (Homoptera: Aphididae). Biodiversitas 12: 187-191.

Huang, W. Y., Y. Z. Cai, J. Xing, H. Corke and M. Sun. 2007. A potential antioxidant resource: Endophytic fungi from medicinal plants. Economic Botany 61: 14-30.

Jiang-Tao, B. I., W. Xiao-Xia, C. Wei-Min, W. Jing and H. E. Da-Han. 2013. Isolation of endophytic fungi from medicinal plant Glycyrrhiza uralensis and its microbial inhibition activity. Pratacultural Science 30: 357-364.

Karliński, L., M. Rudawska, B. Kieliszewska-Rokicka and T. Leski. 2010. Relationship between genotype and soil environment during colonization of poplar roots by mycorrhizal and endophytic fungi. Mycorrhiza 20: 315-324.

Karliński, L., M. Rudawska and T. Leski. 2013. The influence of host genotype and soil conditions on ectomycorrhizal community of poplar clones. European Journal of Soil Biology 58: 51-58.

Kathrin, Blumenstein, David, Macaya-Sanz, Juan, A., Martín, Benedicte, R., Albrectsen. 2015. Phenotype MicroArrays as a complementary tool to next generation sequencing for characterization of tree endophytes. Frontiers in Microbiology 6:1033.

Kivlin, S. N., J. S. Lynn, M. R. Kazenel, K. K. Beals and J. A. Rudgers. 2017. Biogeography of plant " ssociated fungal symbionts in mountain ecosystems: A meta " nalysis. Diversity and Distributions 23: 1067-1077.

Ko D. 2015. Variations in bacterial and fungal community composition along the soil depth profiles determined by pyrosequencing. AGUFM 2015: B11J-0579.

López-Giráldez, F., P. W. Crous, A. Rauhut, D. Hewitt, F. Kauff, W. Untereiner, G. S. D. Hoog, J. P. Townsend, Z. Wang and P. R. Johnston. 2009. The Ascomycota tree of life: a phylum-wide phylogeny clarifies the origin and evolution of fundamental reproductive and ecological traits. -S3. Systematic Biology 58: 224-239.

Li-Ping L, Cui-Ai R, Hong-Yan Z. 2010. Research Progress on Immunomodulatory Effects of Glycyrrhizin. chinese journal of experimental traditional medical formulae 6: 272-276. 
526 Li, B., X. Zhang, F. Guo, W. Wu and T. Zhang. 2013. Characterization of tetracycline

527

528

529

530

531

532

533

534

535

536

537

538

539

540

541

542

543

544

545

546

547

548

549

550

551

552

553

554

555

556

557

558

559

560 resistant bacterial community in saline activated sludge using batch stress incubation with high-throughput sequencing analysis. Water Research 47: 4207-4216.

Li J, Liu S, Wang J, Li J, Li J, Gao W. 2017. Gene expression of glycyrrhizin acid and accumulation of endogenous signaling molecule in Glycyrrhiza uralensis Fisch adventitious roots after Saccharomyces cerevisiae and Meyerozyma guilliermondii applications. Biotechnology and Applied Biochemistry 64: 700-711.

Li Y, Wu H. 2018. The Research Progress of the Correlation Between Growth Development and Dynamic Accumulation of the Effective Components in Medicinal Plants. Chinese Bulletin of Botany 3: 293-304.

Liu, Jinshan, Zhang, Xiang, Wang, Hui, Xiaoli, Zhaohui, Qiu, Weihong. 2018 Long-term nitrogen fertilization impacts soil fungal and bacterial community structures in a dryland soil of Loess Plateau in China. Journal of Soil \& Sediments 18: 1632-1640.

Liu, Wenhong, Zhou, Zhuoyan, Yuhan, Xiufang, Guo, Ying and Junfeng. 2017. Application of High-Throughput Internal Transcribed Spacer rRNA Metagenomics Analysis in Deciphering Endophytic Fungi Diversity of Dendrobium Officinale. Journal of Biobased Materials and Bioenergy 11: 106-118.

Liu, Zhenshan, Wei, Gehong, Yang, Jun, Chen, Weimin, Lin, Yanbing. 2016. Bacterial communities in oil contaminated soils: Biogeography and co-occurrence patterns. Soil Biology \& Biochemistry 98: 64-73.

Liu, Y., Y. Li, W. Luo, S. Liu and G. Wei. 2020. Soil potassium is correlated with root secondary metabolites and root-associated core bacteria in licorice of different ages. Plant and Soil: 1-19.

Martin M. 2011. Cutadapt removes adapter sequences from high-throughput sequencing reads. Embnet Journal 17: 10-12.

Meilan, Chen, Guang, Yang, Ye, Sheng, Pengying, Li, Hongyan, Qiu. 2017. Glomus mosseae Inoculation Improves the Root System Architecture, Photosynthetic Efficiency and Flavonoids Accumulation of Liquorice under Nutrient Stress. Frontiers in Plant Science 8: 931.

Min, J., Chen L, Xin H-L, Zheng C-J, Rahman K, Han T, Qin L-P. 2016. A friendly relationship between endophytic fungi and medicinal plants: a systematic review. Frontiers in microbiology 7: 906.

Mochida K, Sakurai T, Seki H, Yoshida T, Takahagi K, Sawai S, Uchiyama H, Muranaka T, Saito K. 2017. Draft genome assembly and annotation of Glycyrrhiza uralensis, a medicinal legume. The Plant Journal 89: 181-194. 
561 Moricca, S. and A. Ragazzi. 2008. Fungal endophytes in Mediterranean oak forests: a lesson

562

563

564

565

566

567

568

569

570

571

572

573

574

575

576

577

578

579

580

581

582

583

584

585

586

587

588

589

590

591

592

593

594 from Discula quercina. Phytopathology 98: 380-386.

Mylène, H., L. Patricia, G. Julien and H. F. E. Zahar. 2018. Plant host habitat and root exudates shape fungal diversity. Mycorrhiza 28: 451-463.

Ncube, Finnie, JF, Van and Staden. 2012. Quality from the field: The impact of environmental factors as quality determinants in medicinal plants. SAFR J BOT: 11-20.

Nuccio, E. E., J. Anderson-Furgeson, K. Y. Estera, J. Pett-Ridge, P. De Valpine, E. L. Brodie and M. K. Firestone. 2016. Climate and edaphic controllers influence rhizosphere community assembly for a wild annual grass. Ecology 97: 1307-1318.

Orujei, Y., L. Shabani and M. Sharifi-Tehrani. 2013. Induction of glycyrrhizin and total phenolic compound production in licorice by using arbuscular mycorrhizal fungi. Russian Journal of Plant Physiology 60: 855-860.

Palak, Arora, Zahoor, Wani, Tanveer, Ahmad, Phalisteen, Sultan, Suphla. 2019. Community structure, spatial distribution, diversity and functional characterization of culturable endophytic fungi associated with Glycyrrhiza glabra L. Fungal Biology 123: 373-383.

Pimentel IC, Glienke-Blanco C, Gabardo J, Stuart RM, Azevedo JL. 2006. Identification and colonization of endophytic fungi from soybean (Glycine max (L.) Merril) under different environmental conditions. Brazilian Archives of Biology and Technology 49: 705-711.

Pregitzer, K. S., M. J. Laskowski, A. J. Burton, V. C. Lessard and D. R. Zak. 1998. Variation in sugar maple root respiration with root diameter and soil depth. Tree Physiology: 665-670.

Radić, T., M. Likar, K. Hančević, I. Bogdanović and I. Pasković . 2014. Occurrence of root endophytic fungi in organic versus conventional vineyards on the Croatian coast. Agriculture Ecosystems \& Environment 192: 115-121.

Ratnaweera PB, Silva EDD, Williams DE, Andersen RJ. 2015. Antimicrobial activities of endophytic fungi obtained from the arid zone invasive plant Opuntia dillenii and the isolation of equisetin, from endophytic Fusarium sp. BMC Complementary and Alternative Medicine 15:220.

Rizzato, G., E. Scalabrin, M. Radaelli, G. Capodaglio and O. Piccolo. 2017. A new exploration of licorice metabolome. Food Chemistry 221: 959-968.

S., H., Faeth. 2002. Fungal Endophytes: Common Host Plant Symbionts but Uncommon Mutualists. Integrative and Comparative Biology 42:360-368. 
595

596

597

598

599

600

601

602

603

604

605

606

607

608

609

610

611

612

613

614

615

616

617

618

619

620

621

622

623

624

625

626

627

628

Saikkonen, K., S. H. Faeth, M. Helander and T. J. Sullivan. 1998. FUNGAL ENDOPHYTES: A Continuum of Interactions with Host Plants. Annual Review of Ecology \& Systematics 29: 319-343.

Saikkonen, K., P. Wäli, M. Helander and S. H. Faeth. 2004. Evolution of endophyte-plant symbioses. Trends in Plant Science 9: 275-280.

Saude, C., O. P. Hurtado-Gonzales, K. H. Lamour and M. K. Hausbeck. 2008. Occurrence and characterization of a Phytophthora sp. pathogenic to asparagus (Asparagus officinalis) in Michigan. Phytopathology 98: 1075-1083.

Schröfelbauer, B., J. Raffetseder, M. Hauner, A. Wolkerstorfer, W. Ernst and O. H. J. Szolar. 2009. Glycyrrhizin, the main active compound in liquorice, attenuates proinflammatory responses by interfering with membrane-dependent receptor signalling. Biochemical Journal 421: 473-482.

Shah, A., M. A. Rather, A. M. Shah, S. Mushtaq, A. Hussain, S. Rasool, Z. A. Parry and P. H. Qazi. 2016. Evaluating the in vitro antituberculosis, antibacterial and antioxidant potential of fungal endophytes isolated from Glycyrrhiza glabra L. Annals of Phytomedicine 5: 140-146.

Singh G, Mukerji KG. 2006. Root Exudates as Determinant of Rhizospheric Microbial Biodiversity. Microbial Activity in the Rhizoshere 7: 39-53.

Stegen JC, Lin X, Konopka AE, Fredrickson JK. 2012. Stochastic and deterministic assembly processes in subsurface microbial communities. Isme Journal 6:1653-1664.

Stephenson SL, Tsui C, Rollins AW. 2013. Methods for Sampling and Analyzing Wetland Fungi: Springer Netherlands 93-121.

Tan X, Zhou Y, Zhou X, Xia X, Wei Y, He L, Tang H, Yu L. 2018. Diversity and bioactive potential of culturable fungal endophytes of Dysosma versipellis; a rare medicinal plant endemic to China. Scientific Reports 8: 1-9.

Taylor DL, Hollingsworth TN, Mfarland JW, Lennon NJ, Nusbaum C, Ruess RW. 2014. A first comprehensive census of fungi in soil reveals both hyperdiversity and fine-scale niche partitioning. Ecological Monographs 84: 3-20.

Tianshui Niu JY, Long Zhang, Xiao Cheng, Kai Li, Gang Zhou. 2009. Research Advances on Anticancer Effect of Licorice. Current Bioactive Compounds 5: 234-242.

Tie, W., Y. Hu, J. Zhu and Z. Lin. 2010. Isolation and i dentification of endophytical fungus in Licorice collected from Xinjiang area. Biotechnology bulletin 9: 149-153.

Trail, F. 2010. Fungal cannons: explosive spore discharge in the Ascomycota. Fems Microbiology Letters 276: 12-18. 
629 Vaz ABM, Mota RC, Bomfim MRQ, Vieira MLA, Zani CL, Rosa CA, Rosa LH. 2009.

630 Antimicrobial activity of endophytic fungi associated with Orchidaceae in Brazil.

631 Canadian Journal of Microbiology 55: 1381-1391.

632 Wallen, R. M. and M. H. Perlin. 2018. An overview of the function and maintenance of sexual

633 reproduction in dikaryotic fungi. Frontiers in microbiology 9: 503.

634 Wang L, Yang R, Yuan B, Liu Y, Liu C. 2015. The antiviral and antimicrobial activities of

635 licorice, a widely-used Chinese herb. Acta Pharmaceutica Sinica B 5:310-315.

636 Wang, Y. and W. H. Wu. 2017. Regulation of potassium transport and signaling in plants.

637 Current Opinion in Plant Biology 39: 123-128.

638 Weidner, S., E. Kordala, W. Brosowska-Arendt, M. Karamac, A. Kosinska and R.

639

640

641 Amarowicz. 2009. Phenolic compounds and properties of antioxidants in grapevine roots

642 (Vitis vinifera L.) under drought stress followed by recovery. Acta Societatis Botanicorum Poloniae 78: 279-286.

643

644

Xie W, Hao Z, Yu M, Wu Z, Chen B. 2019. Improved phosphorus nutrition by arbuscular

645

mycorrhizal symbiosis as a key factor facilitating glycyrrhizin and liquiritin accumulation in Glycyrrhiza uralensis. Plant and Soil 439: 243-257.

648

649

650

651

652

653

654

655

656

657

658

659

660

Winkel-Shirley, B. 2002. Biosynthesis of flavonoids and effects of stress. Current Opinion in Plant Biology 5: 218-223.

Yina, L., E. G. a, Y. Z. a, Z. S. a and W. Bing. 2018. Chemical Profile and Anti-inflammatory Activity of Total Flavonoids from Glycyrrhiza Uralensis Fisch. Iranian Journal of Pharmaceutical Research Ijpr 17: 726-734.

Zhang D, Wang H. 2005. Preliminary Study on the Growth Pattern of Several Clonal Plants in Desert Zones of Xinjiang. Arid Zone Research 22: 219-224.

Zhang, J., C. Wang, S. Guo, J. Chen and P. Xiao. 1999. Studies on the plant hormones produced by 5 species of endophytic fungi isolated from medicinal plants (Orchidacea). Zhongguo yi xue ke xue Yuan xue bao. Acta Academiae Medicinae Sinicae 21: 460-465.

Zhang, Y., Y. Yang, H. Gong and H. Zhu. 2018. A systematic review of the comparison of three medicinal licorices, based on differences of the types and contents about their bioactive components. Journal of Chemical Biology and Pharmaceutical Chemistry 1: 16.

Zhao, Shan, Mou and Zhou. 2011. Plant-Derived Bioactive Compounds Produced by Endophytic Fungi. MINI-REV MED CHEM 11: 159-168. 


\section{Table $\mathbf{1}$ (on next page)}

Pearson correlation coefficient of the content of bioactive compounds with soil physicochemical properties

the values are Pearson's correlation coefficients (significance level was set at 0.05 ). The correlation coefficient $r$ of Pearson is between -1 and $1, r<0$ means negative correlation, $r\rangle$ 0 means positive correlation, $\mathrm{r}=0$ means no linear correlation. ** means $\mathrm{P}<0.01$; * means $P<0.05$. Abbreviations: GIA, glycyrrhizic acid; GTF, total flavonoid; LI, liquiritin; SOM, soil organic matter; STN, soil total nitrogen; STP, soil total phosphorus; STK, soil total potassium; SNN, soil nitrate nitrogen; SAN, soil ammonium nitrogen; SAP, soil available phosphorus; SAK, soil available potassium; TS, total salt; PH, soil pH; SWC, soil water content. 
Table 1. Pearson correlation coefficient of the content of bioactive compounds with soil physicochemical properties

\begin{tabular}{|c|c|c|c|c|c|c|c|c|c|c|c|c|c|c|}
\hline & GIA & GTF & LI & SOM & STN & TP & STK & SNN & SAN & ;AP & AK & TS & PH & swc \\
\hline GIA & 1.000 & * & 0.294 & -0.146 & 0.121 & 0.158 & -0.107 & -0.144 & -0.337 & -0.121 & $0.463^{*}$ & -0.069 & 0.263 & $0.609^{* *}$ \\
\hline GTF & $0.419^{*}$ & 1.000 & 0.172 & -0.142 & 0.132 & 0.345 & -0.121 & 0.048 & -0.008 & 0.034 & וטא. & -0.217 & 0.000 & 0.160 \\
\hline $\mathrm{LI}$ & 0.294 & 0.172 & 1.000 & -0.031 & 0.183 & 0.251 & -0.294 & 0.070 & 0.239 & -0.216 & $-0.415^{\star}$ & $-0.403^{*}$ & 0.058 & -0.183 \\
\hline $\begin{array}{l}\text { SO } \\
M\end{array}$ & -0.146 & -0.142 & -0.031 & 1 & 0.274 & ** & 0 & & 0 & & 6 & 36 & 99 & -0.327 \\
\hline STN & 0.121 & 0.132 & 0.183 & 0.274 & 1.000 & 0.4 & -0.249 & 0.4 & 0. & 0.4 & -0.022 & 0.166 & -0.236 & -0.300 \\
\hline STP & 0.158 & 0.345 & 0.251 & $-0.527^{\star *}$ & $\begin{array}{c}0.455 \\
*\end{array}$ & 1.000 & $-0.465^{*}$ & -0.198 & 0.245 & -0.045 & -0.034 & -0.119 & 0.090 & -0.033 \\
\hline STK & -0.107 & -0.121 & -0.294 & 0.238 & -0.249 & $-0.465^{\star}$ & 1.000 & 0.167 & -0.326 & 0.211 & 0.247 & 0.182 & -0.020 & 0.156 \\
\hline SNN & -0.144 & 0.048 & 0.070 & $0.532^{* *}$ & $\begin{array}{c}0.416 \\
*\end{array}$ & -0.198 & 0.167 & 1.000 & 0.267 & $0.736^{* *}$ & -0.011 & $0.489^{* *}$ & -0.284 & $-0.411^{*}$ \\
\hline SAN & -0.337 & -0.008 & 0.239 & 0.248 & 0.333 & 0.245 & -0.326 & 0.267 & 1.000 & 0.076 & $-0.566^{\star *}$ & -0.066 & -0.315 & $-0.641^{* *}$ \\
\hline SAP & -0.121 & 0.034 & -0.216 & $0.400^{*}$ & $\begin{array}{c}0.415 \\
*\end{array}$ & -0.045 & 0.211 & $0.736^{* *}$ & 0.076 & 1.000 & 0.364 & 0.7 & -0.253 & -0.172 \\
\hline SAK & $0.463^{*}$ & 0.151 & $-0.415^{*}$ & -0.176 & -0.022 & -0.034 & 0.247 & -0.011 & $-0.566^{* *}$ & 0.364 & 1.000 & $0.556^{* *}$ & 0.063 & $0.750^{* *}$ \\
\hline TS & -0.069 & -0.217 & $-0.403^{*}$ & 0.136 & 0.166 & -0.119 & 0.182 & $0.489^{* *}$ & -0.066 & $0.723^{* *}$ & $0.556^{\star *}$ & 1.000 & -0.062 & 0.238 \\
\hline $\mathrm{PH}$ & 0.263 & 0.000 & 0.058 & -0.229 & -0.236 & 0.090 & -0.020 & -0.284 & -0.315 & -0.253 & 0.063 & -0.062 & 1.000 & $0.446^{*}$ \\
\hline $\begin{array}{l}\text { SW } \\
\text { C }\end{array}$ & $0.609^{* *}$ & 0.160 & -0.183 & -0.327 & -0 & -0.033 & 56 & $-0.411^{*}$ & -0.6 & 72 & ** & 0.238 & $\begin{array}{c}0.446 \\
*\end{array}$ & 1.000 \\
\hline
\end{tabular}

2 Description: the values are Pearson's correlation coefficients (significance level was set at 0.05 ). The correlation coefficient $r$ of Pearson is between

$3-1$ and $1, r<0$ means negative correlation, $r>0$ means positive correlation, $r=0$ means no linear correlation. ${ }^{* *}$ means $P<0.01 ;{ }^{*}$ means $\mathrm{P}<0.05$. 
4 Abbreviations: GIA, glycyrrhizic acid; GTF, total flavonoid; LI, liquiritin; SOM, soil organic matter; STN, soil total nitrogen; STP, soil total phosphorus;

5 STK, soil total potassium; SNN, soil nitrate nitrogen; SAN, soil ammonium nitrogen; SAP, soil available phosphorus; SAK, soil available potassium;

6 TS, total salt; $\mathrm{PH}$, soil $\mathrm{pH}$; SWC, soil water content. 
Figure 1

Effect of main effect plant species on the effective ingredients of licorice roots

Ordinate is the content of liquiritin (a), glycyrrhizic acid (b) and total flavonoid (c); abscissa is the group name: $\mathrm{Gi}, \mathrm{Gg}$ and $\mathrm{Gu}$ : Glycyrrhiza inflata, Glycyrrhiza glabra and Glycyrrhiza uralensis, and different letters indicated significance test $(p<0.05)$. Bars (mean with standard error) with different lowercase letters indicated significant difference $(P<0.05)$ assessed by one-way analysis of variance followed by Bonferroni's test for multiple comparisons.

a

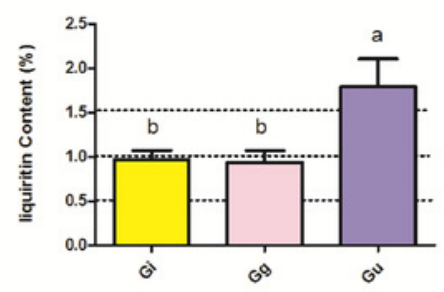

b

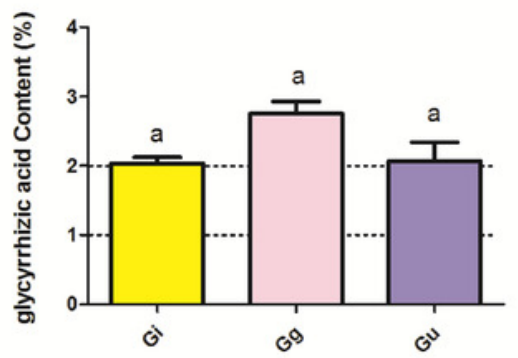

C

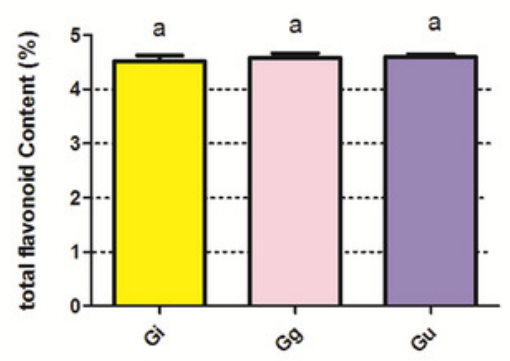




\section{Figure 2}

The significance test of the differences of Alpha Diversity

Ordinates are Shannon index (a) and Chaol index (b), respectively. Abscissa is the group name: Gi, Gg and Gu: Glycyrrhiza inflata, Glycyrrhiza glabra and Glycyrrhiza uralensis; 1, 2 and 3: root depth $0-20 \mathrm{~cm}, 20-40 \mathrm{~cm}$, and $40-60 \mathrm{~cm}$, respectively. The mark * is significance test $p<0.05$.

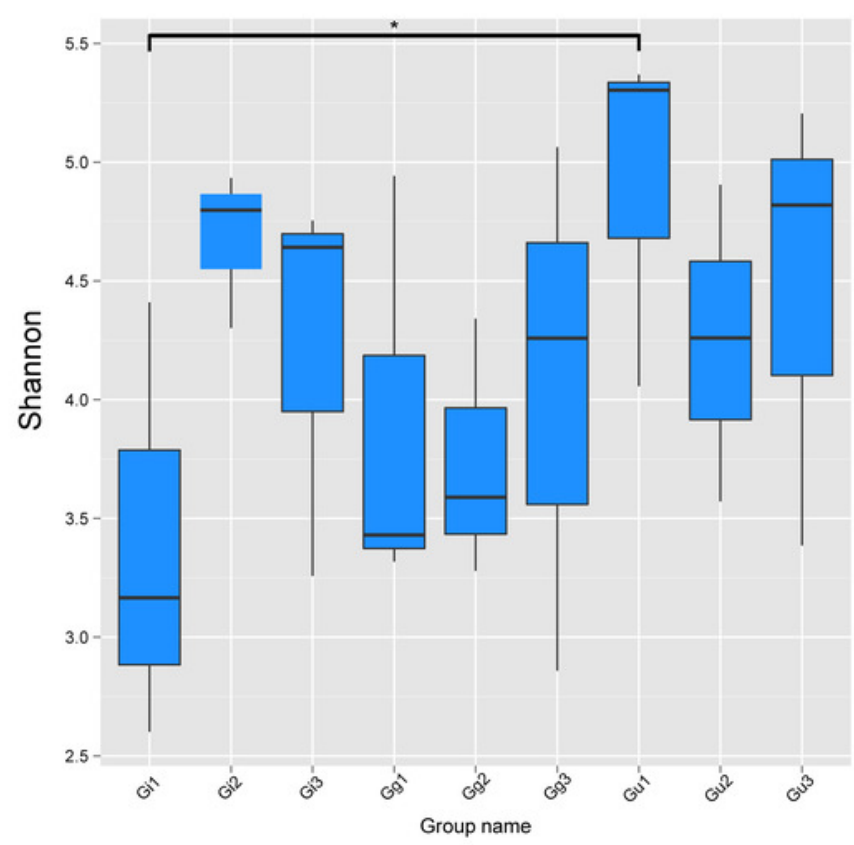

b

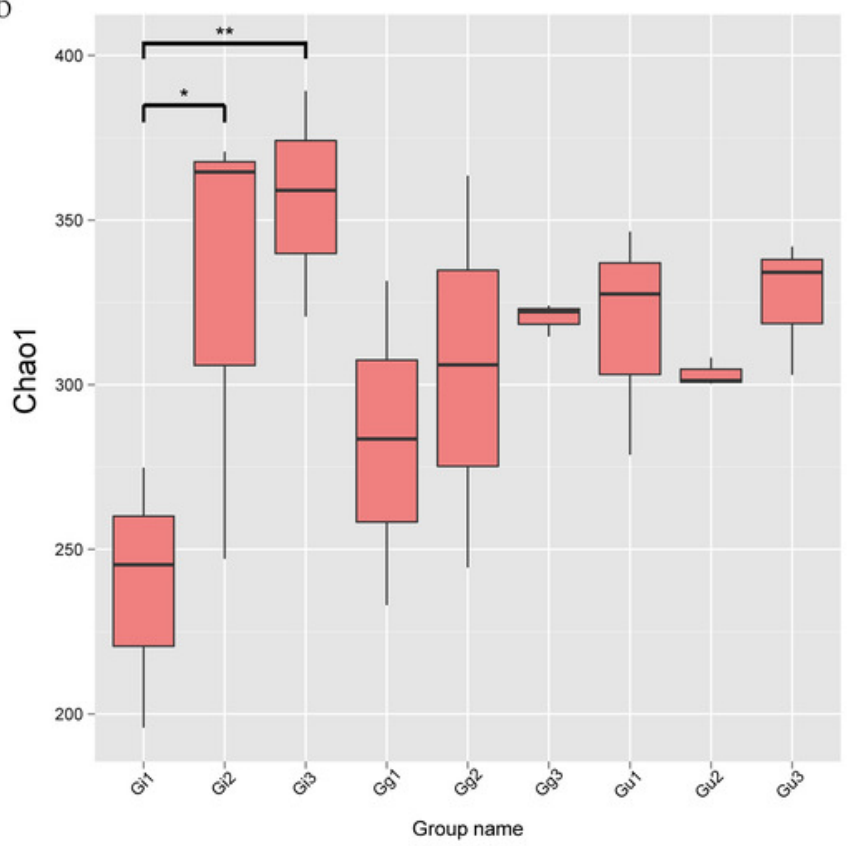




\section{Figure 3}

Unweighted Pair-Group Method with Arithmetic (UPGMA) clustering tree base on the weighted unifrac distance (a); and the significance test of the differences of Beta Diversity (b).

a: The left is the UPGMA cluster tree structure, and the right is the distribution of relative abundance of each sample at the phylum level; $b$ : Ordinate is the Beta diversity; Abscissa is the group name: $\mathrm{Gi}, \mathrm{Gg}$ and $\mathrm{Gu}$ : Glycyrrhiza inflata, Glycyrrhiza glabra and Glycyrrhiza uralensis; 1,2 and 3: root depth $0-20 \mathrm{~cm}, 20-40 \mathrm{~cm}$, and $40-60 \mathrm{~cm}$, respectively. The mark * is significance test $p<0.05$. 
a

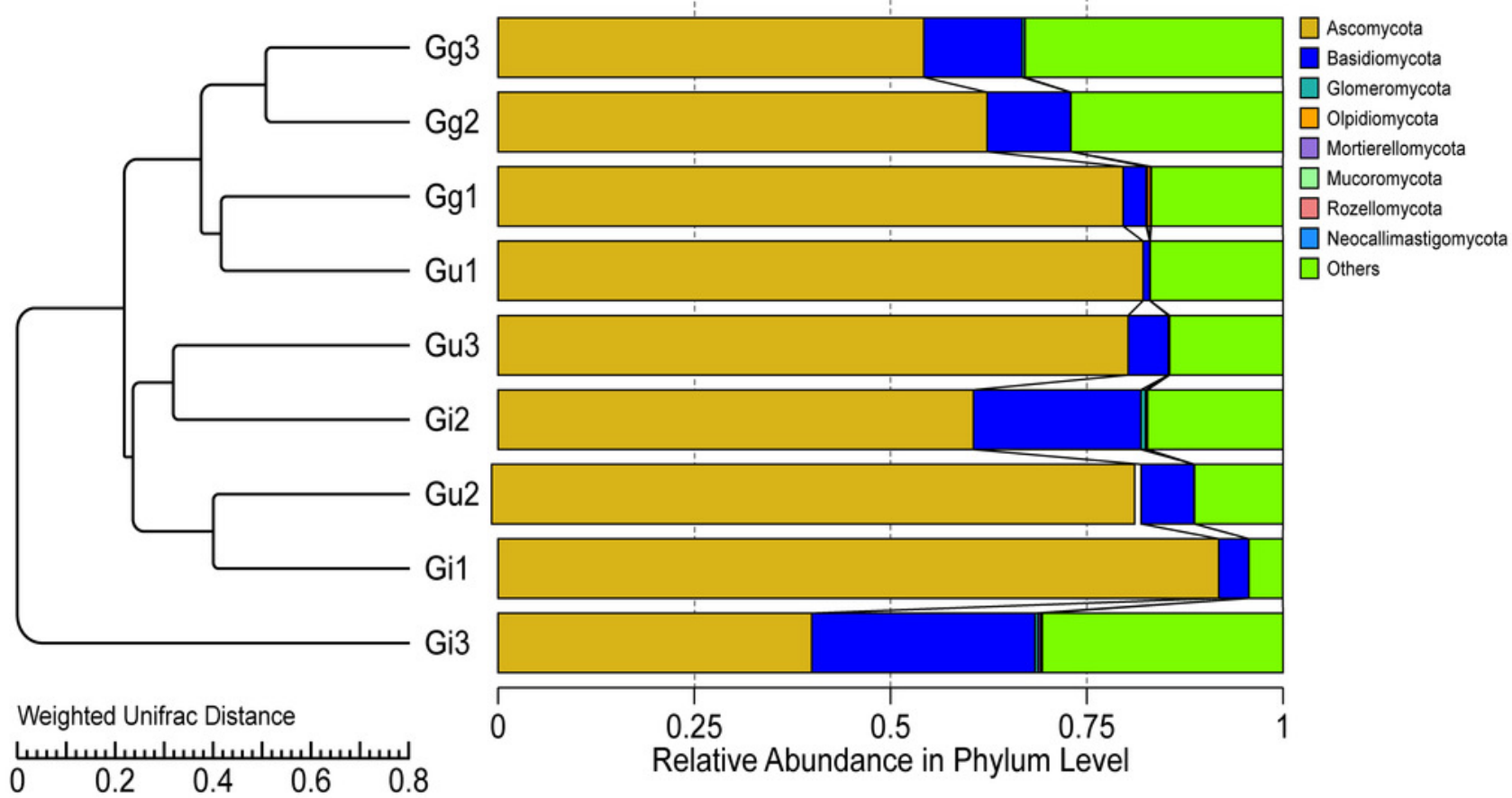

b

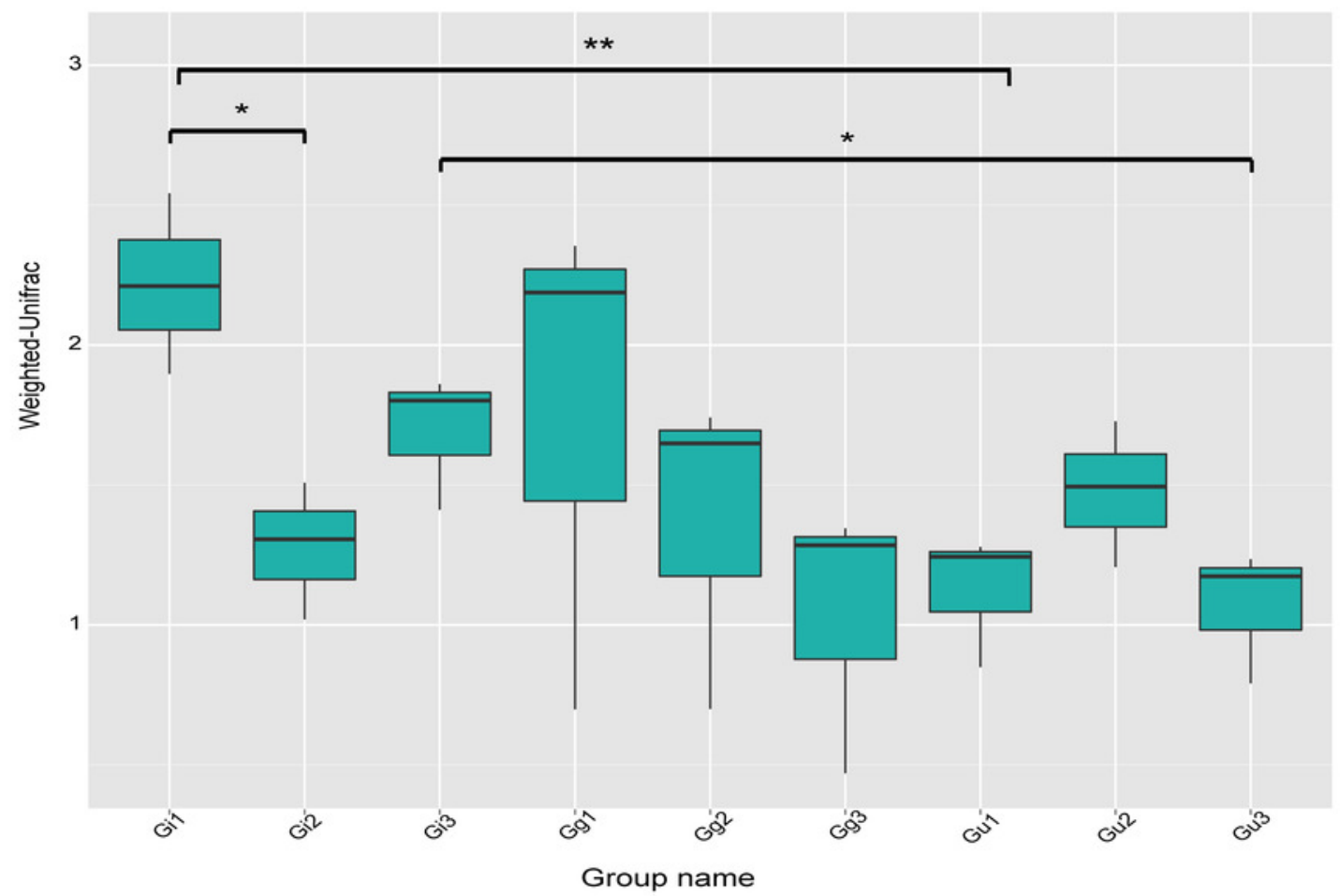


Figure 4

Histograms of relative abundance of the top 10 endophytic fungi at the phyla (a) level of taxonomy and difference analysis at the Phylum classification level (b), Histograms of relative abundance of the top 10 endophytic fungi at the genera (c) level.

Ordinate is the relative abundance; others refers to are sequences with less or not be annotated. Abscissa is the group name: Gi, Gg and Gu:Glycyrrhiza inflata,Glycyrrhiza glabra and Glycyrrhiza uralensis; 1, 2 and 3: root depth $0-20 \mathrm{~cm}, 20-40 \mathrm{~cm}$, and $40-60 \mathrm{~cm}$, respectively. $* *$ means $\mathrm{P}<0.01$.

a
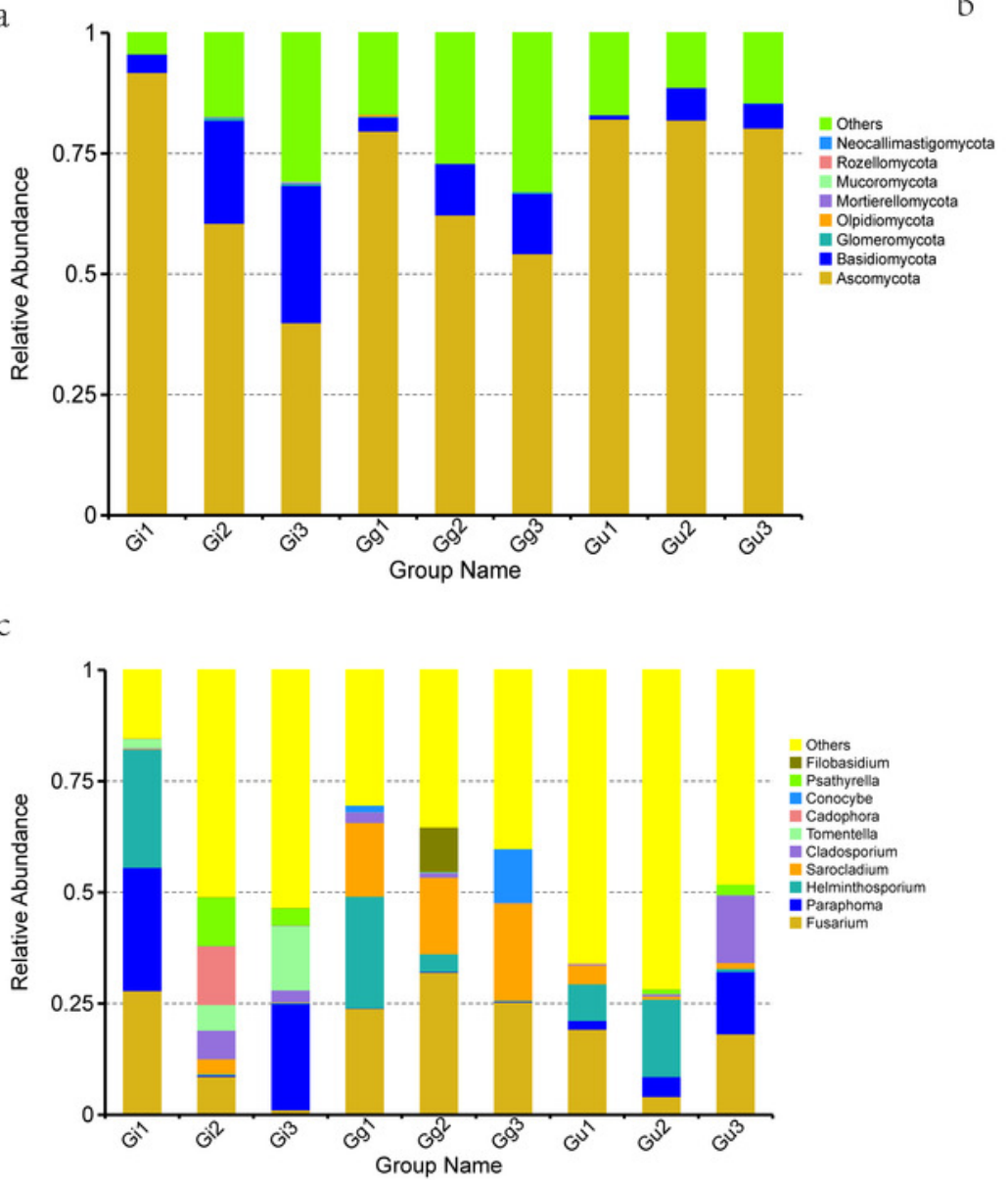

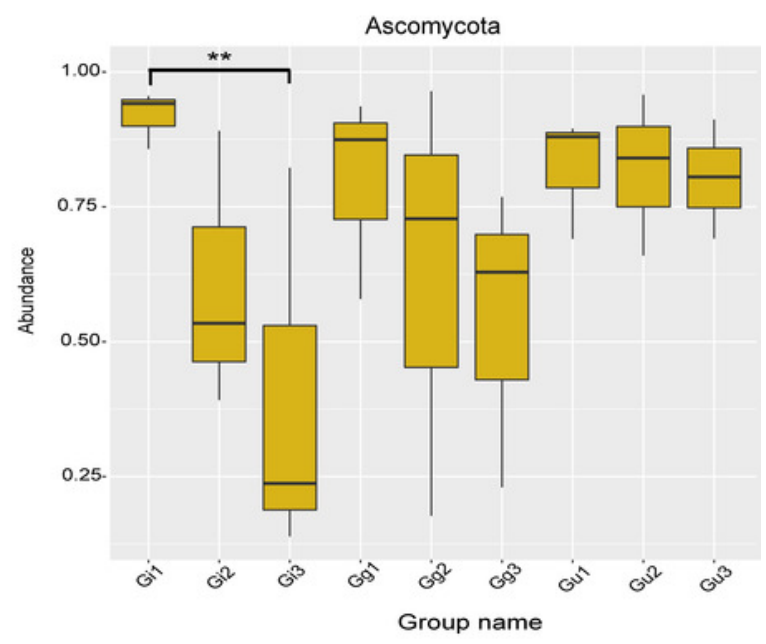




\section{Figure 5}

\section{Heatmaps of Spearman correlation analysis}

Ordinate is the information of environmental factors, and abscissa is the information of alpha diversity indexes. The correlation coefficient $r$ of Spearman is between -1 and $1, r<0$ is negative correlation, $r>0$ is positive correlation, and the mark * is significance test $(p<$ 0.05). Abbreviations: SOM, soil organic matter; STN, soil total nitrogen; STP, soil total phosphorus; STK, soil total potassium; SNN, soil nitrate nitrogen; SAN, soil ammonium nitrogen; SAP, soil available phosphorus; SAK, soil available potassium; TS, total salt; PH, soil pH; SWC, soil water content; RWC, root water content; GIA, glycyrrhizic acid; GTF, total flavonoid; LI, liquiritin. 
Spearman Correlation Heatmap

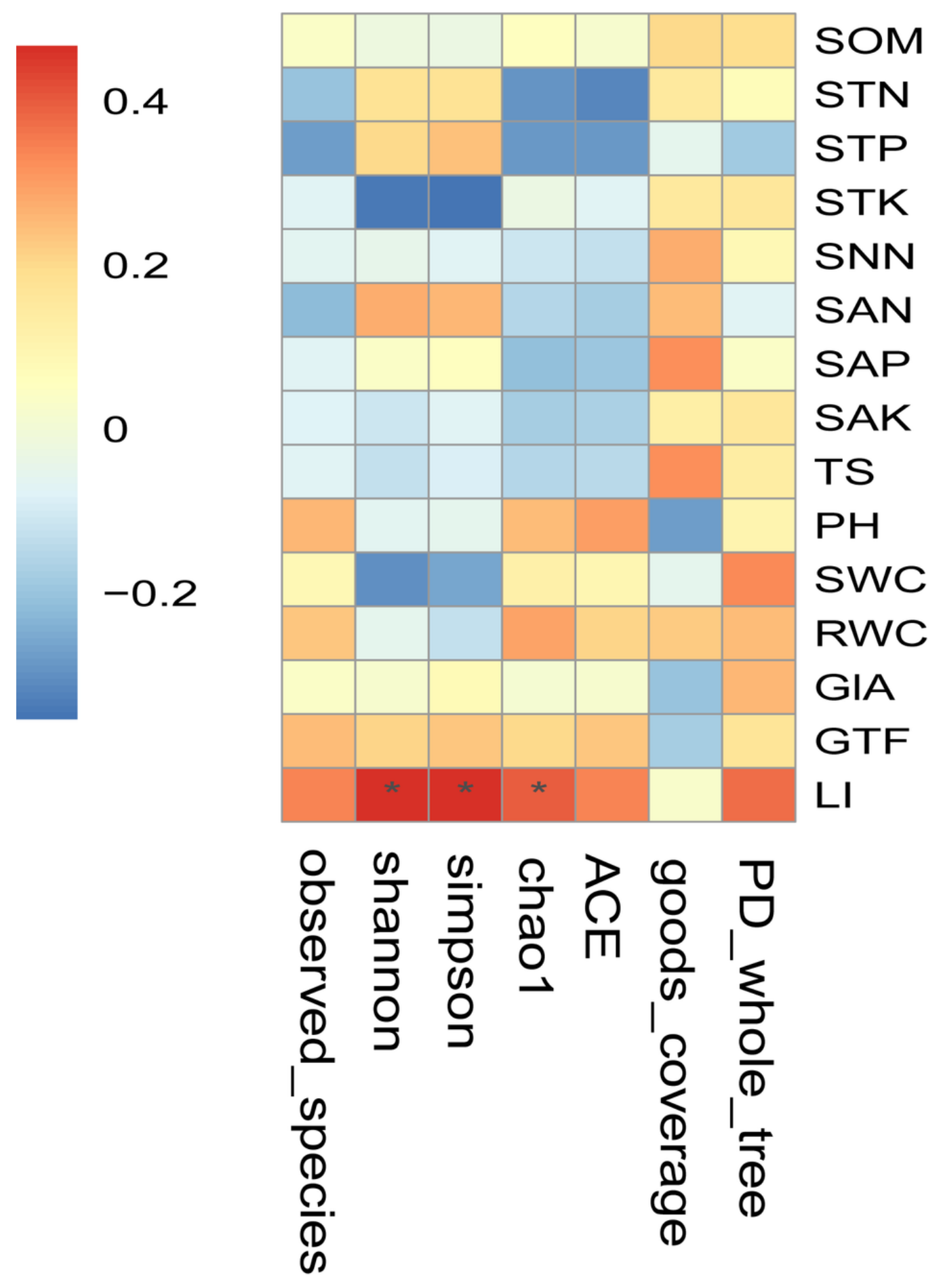




\section{Figure 6}

Distance-based redundancy analysis (db-RDA) for all groups

Environmental factors are generally represented by arrows. The length of the arrow line represents the degree of correlation between a certain environmental factor and community and species distribution, and the longer the arrow, the greater the correlation. When the angle between the environmental factors is acute, it means that there is a positive correlation between the two environmental factors, while when the angle is obtuse, there is a negative correlation. Abbreviations: SOM, soil organic matter; STN, soil total nitrogen; STP, soil total phosphorus; STK, soil total potassium; SNN, soil nitrate nitrogen; SAN, soil ammonium nitrogen; SAP, soil available phosphorus; SAK, soil available potassium; TS, total salt; PH, soil pH; SWC, soil water content; RWC, root water content; GIA, glycyrrhizic acid; GTF, total flavonoid; LI, liquiritin. Gi, Gg and Gu: Glycyrrhiza inflata, Glycyrrhiza glabra and Glycyrrhiza uralensis; 1, 2 and 3: root depth $0-20 \mathrm{~cm}, 20-40 \mathrm{~cm}$, and $40-60 \mathrm{~cm}$, respectively. 


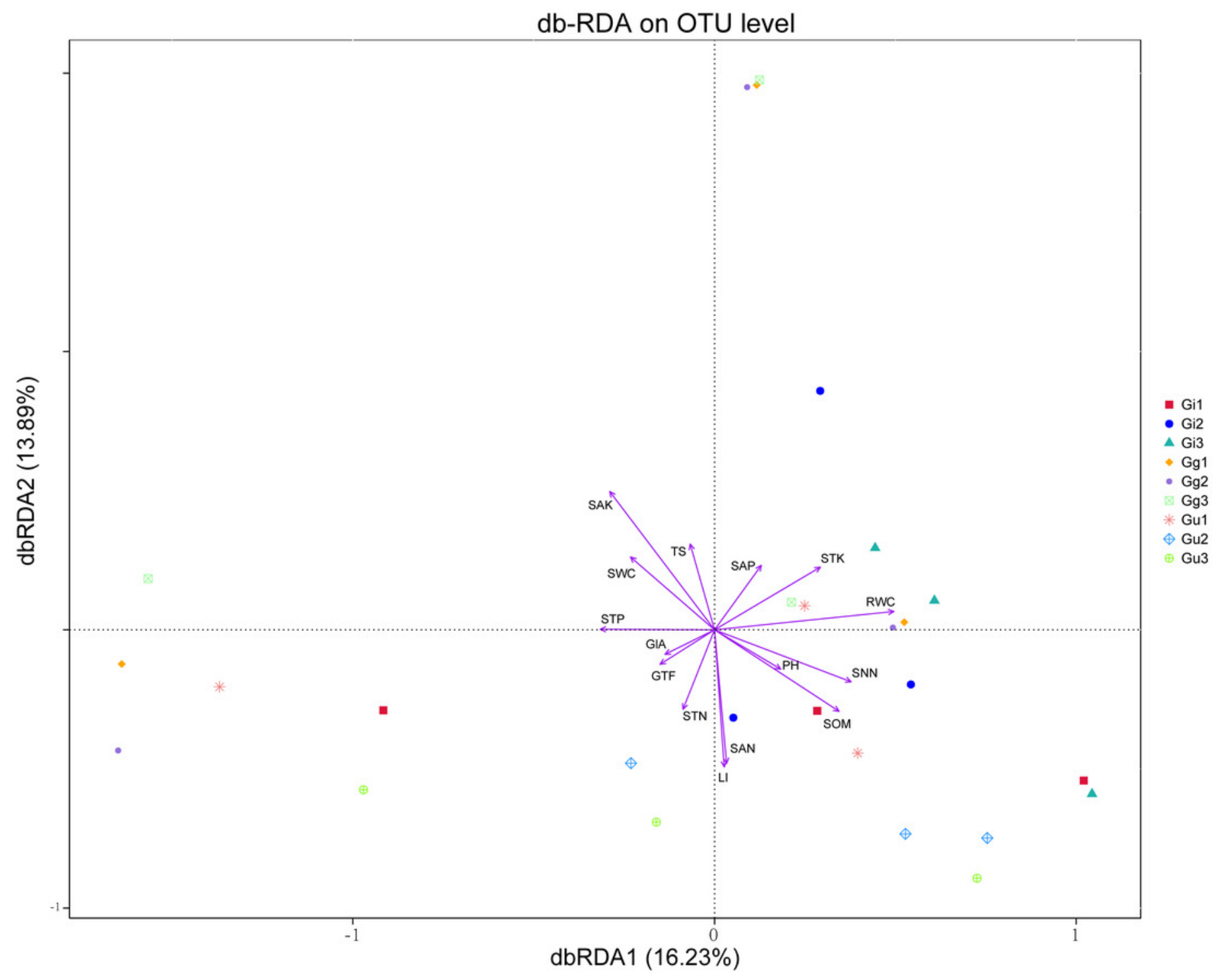

